\title{
Psychometric properties of Persian version of Doctor-Patient Communication (DPC) Scale for skill assessment in acute and critical conditions in Iran
}

\author{
Maryam Akbarilakeh $^{1 *}$, Simin Shouaybi $^{2}$ \\ 1. Center for Educational Research in Medical Sciences (CERMS), School of Medicine, Iran University of Medical Sciences, \\ Tehran, Iran \\ 2. Virtual School of Medical Education and Management, Shahid Beheshti University of Medical Sciences, Tehran, Iran
}

Received: 16 February 2021

Accepted for publication: 14 September 2021

[EPub a head of print-25 September 2021]

Payesh: 2021; 20 (5): 619- 628

\begin{abstract}
Objective(s): Establishing a proper relationship between patient and physician is an essential component of quality medical care and effective communication plays an important role in health system. This study was conducted to investigate the psychometric properties of the DPC for doctor-patient communication skill assessment scale in acute and critical conditions in Iran.

Methods: In this study, the Persian version of the physician-patient relationship assessment scale in acute and critical conditions was used. The statistical population included patients referring to the hospitals affiliated to the Shahid Beheshti University of Medical Sciences. Based on the inclusion criteria, $(n=380)$ were selected by convenience sampling method. After translation and adaptation; face and content validity, reliability and structural validity (confirmatory factor analysis) were used.

Results: Out of 380 participants in the study, $233(61.3 \%)$ were female and 147 (38.7\%) were male. More than half of participants $198(52.1 \%)$ had a secondary education and $315(82.9 \%)$ were employed. The content validity ratio according to experts' panel was 0.99 . Confirmatory factor analysis showed that of all 15 items were loaded with a factor loading above 0.4 and the scale had a good fit in Iranian society. Reliability was 0.96 using Cronbach's alpha and 0.9 using test retest analysis.

Conclusion: The Iranian version of doctor-patient communication for skill assessment in acute and critical conditions showed good validity and reliability. By using the scale health system managers can recognize the strengths and weaknesses of communication skill and based on, they can design appropriate interventions.
\end{abstract}

Key words: Psychometrics, communication skills, acute and critical conditions

\footnotetext{
* Corresponding author: School of Medicine, Iran University of Medical Sciences, Tehran, Iran

E-mail: akbari_maryam59@yahoo.com
} 


\title{
روانسنجى نسخه فارسى مقياس ارتباط يزشك و بيمار (DPC) براى ارزيابى مهارت در مواقع حاد و بحرانى در ايران
}

\author{
مريم اكبرى لاكه'، سيمين شعيبى \\ ا. مركز تحقيقات آموزش علوم يزشكى، دانشكده يزشكى، دانشكاه علوم يزشكى ايران، تهران، ايران

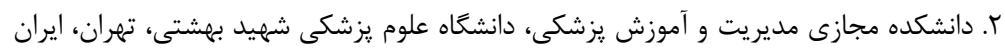

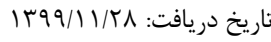

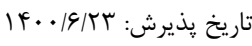

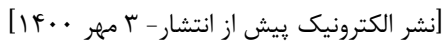

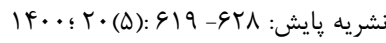

جكيده

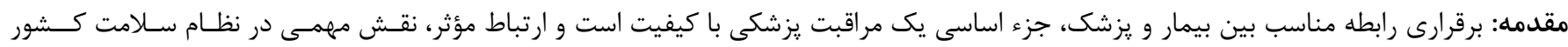

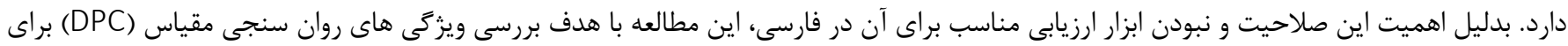

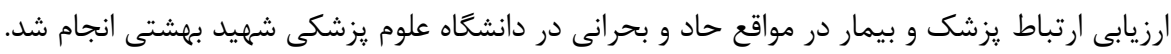

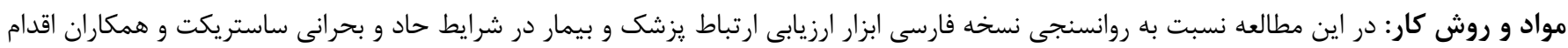

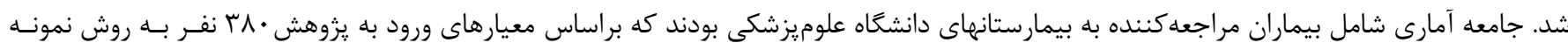

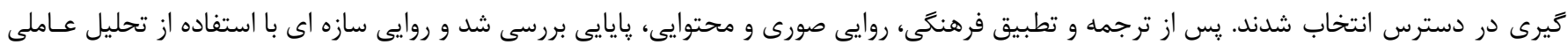

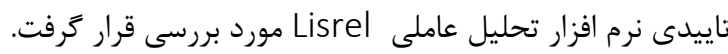

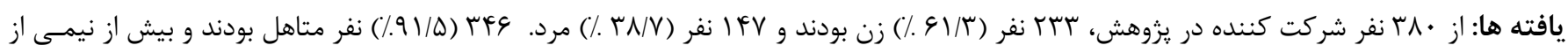

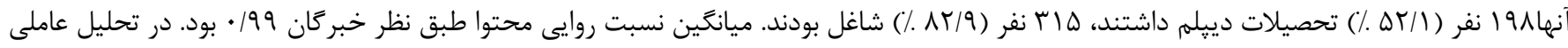

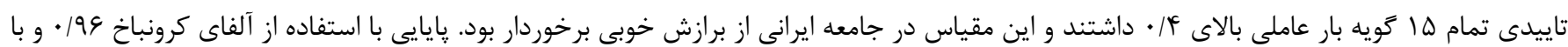
روش آزمون باز آزمون، 9 • • بود.

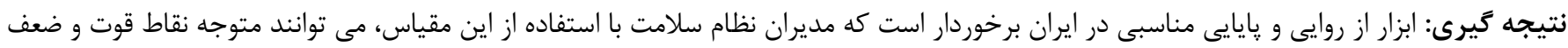

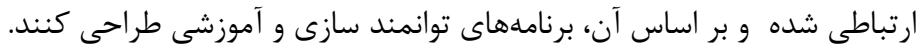

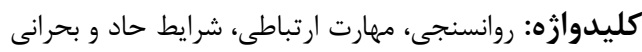

كداخلاق: IR.SBMU.SME.REC.1398.12 
بصورت يكيارجه و منسجم در جهت كاهش آثار و عواقب بحران و يا

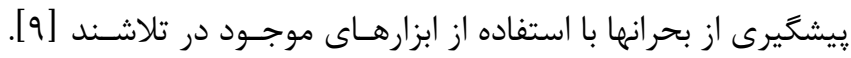

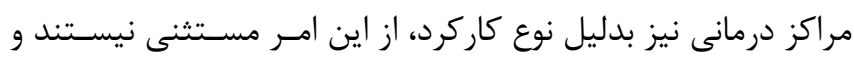

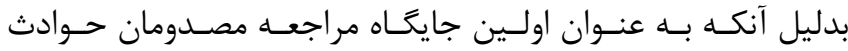

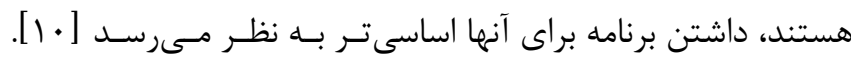

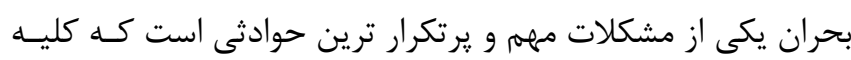

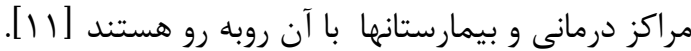

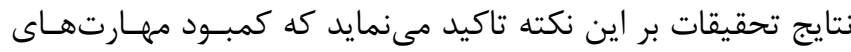

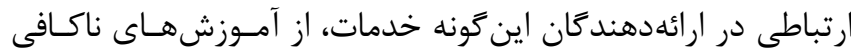

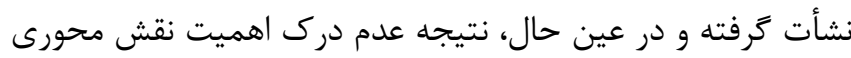

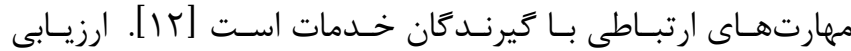

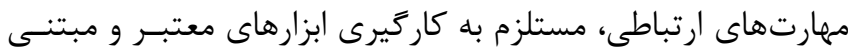

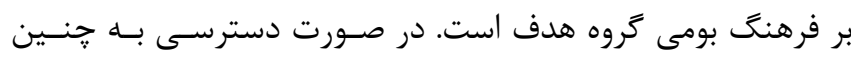

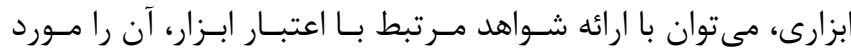

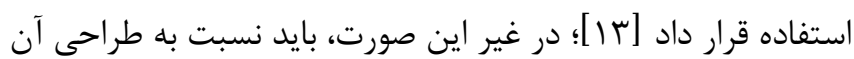

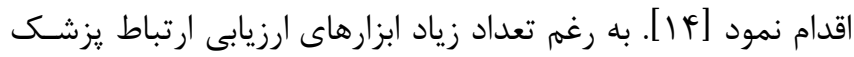

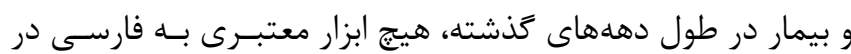

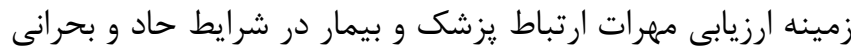

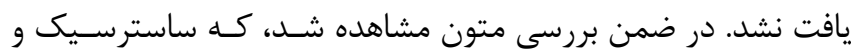

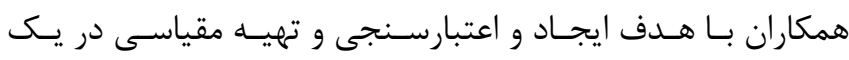

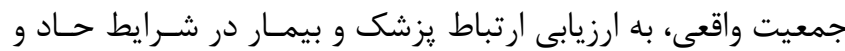
بحرانى مبتنى بر بررسى مرور نظام مند يرداختند و مقيساس(DPC)

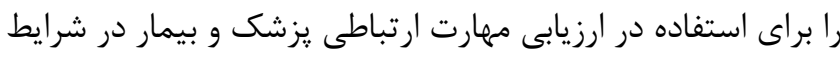

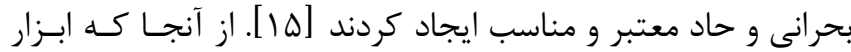

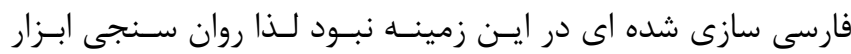

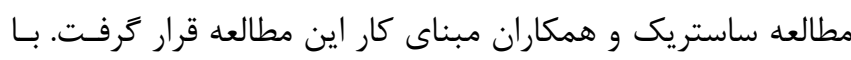
توجه به اينكه تفاوت در تعاريف ابعادى مختلف در علـوم يزشـكى و و

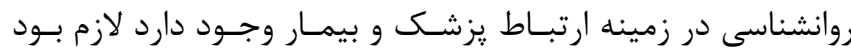

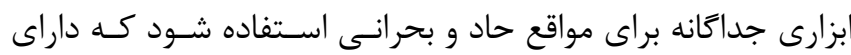

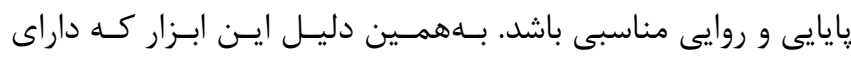

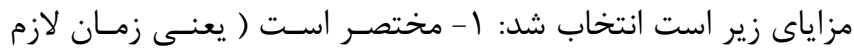

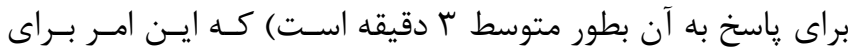

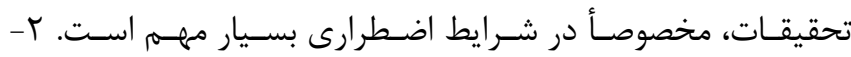

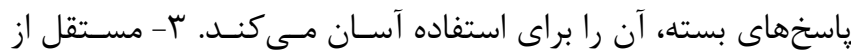

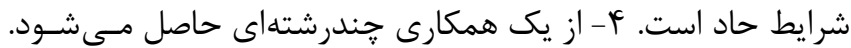
ه- داراى همبستگى داخلى خوبى است و مبتنى بر يك مدل نظرى

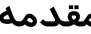

ارتباط يكى از اساسىترين صلاحيت هاى يزشكان است كه هميشـهـ

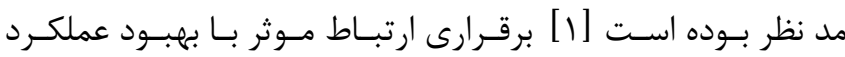

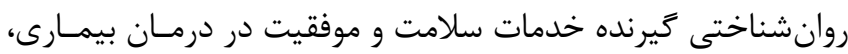

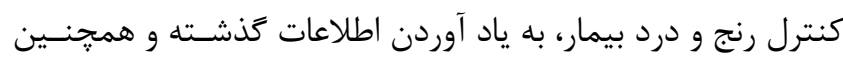

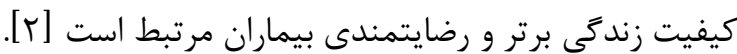

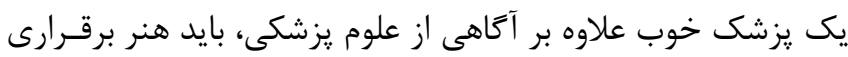

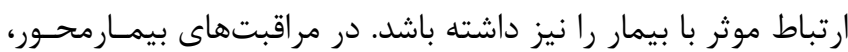

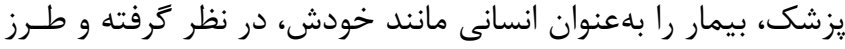

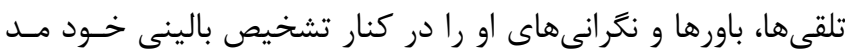

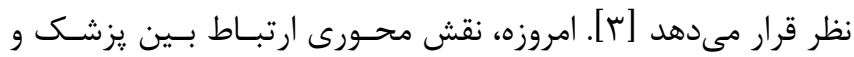

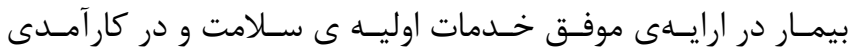

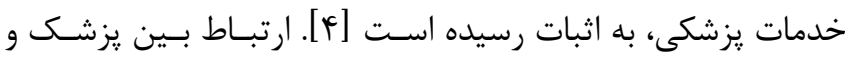

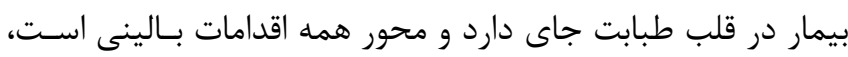

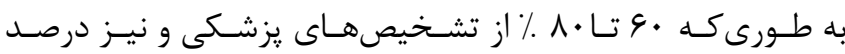

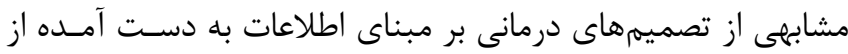

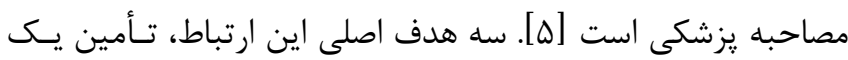

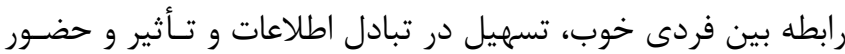

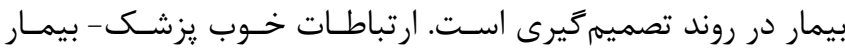

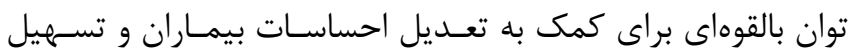

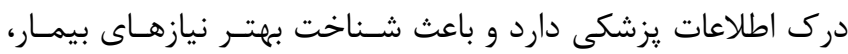

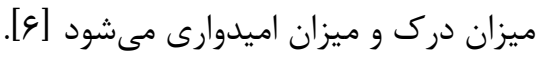

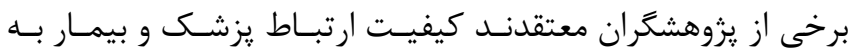

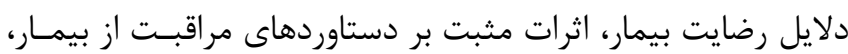

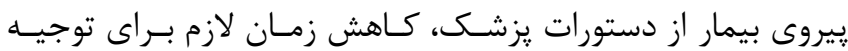

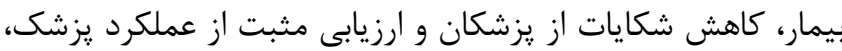

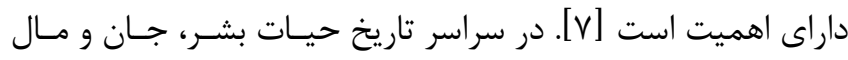

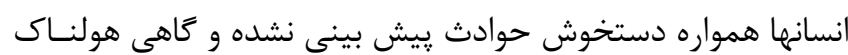

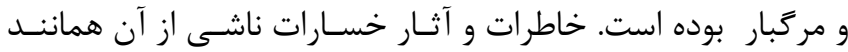

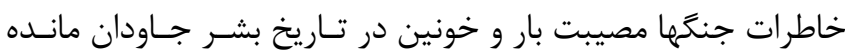

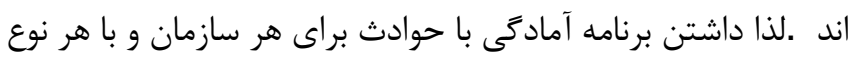

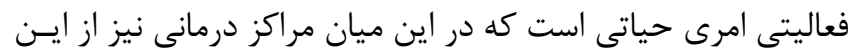
قاعده مستثنى نيستند و حتى بدليل جايخـاه حسـاس آنهـا در امـر

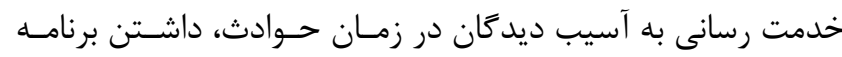

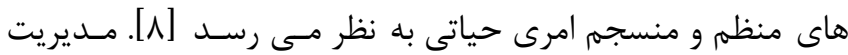

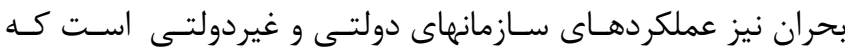


ارتباط ماما- مادر در اتاق زايمان است [ [Y]]. وكيلى و همكاران نيـز شواهد مناسبى در خصوص استحكام ساختار عـاملى و وِايـايى ابـزار

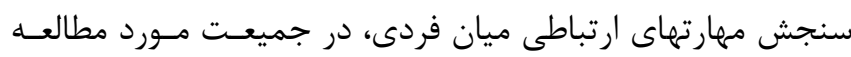

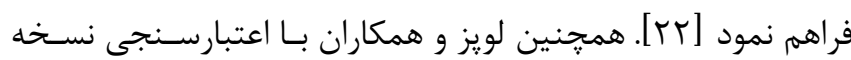

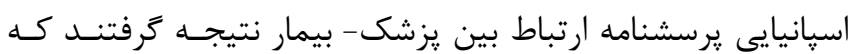

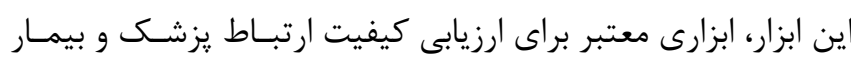

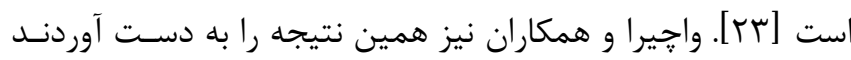

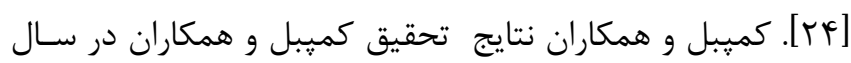

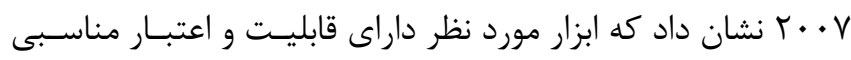

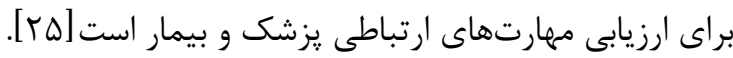

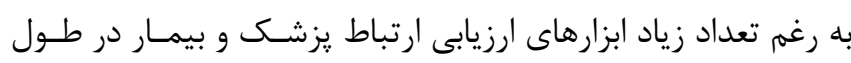

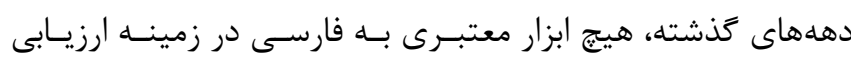

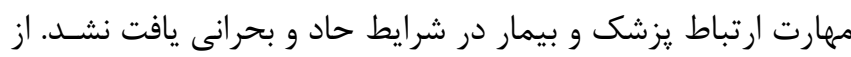

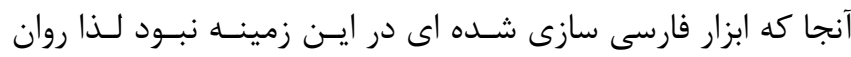

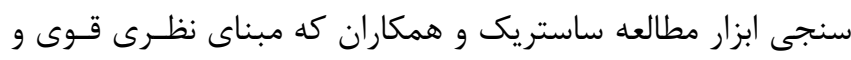

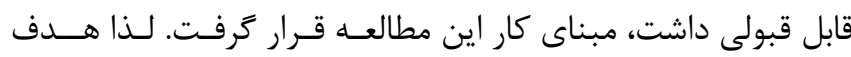

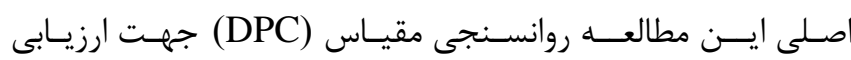

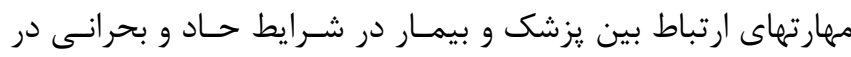

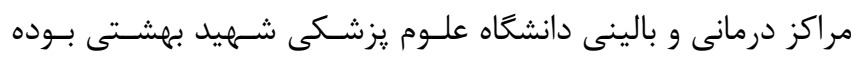

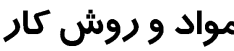

اين مطالعه روانسنجى با دو رويكرد توصيفى و تحليلى انجام شد كه

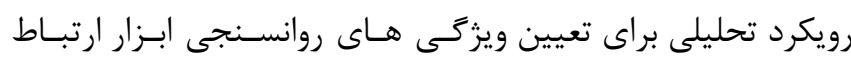

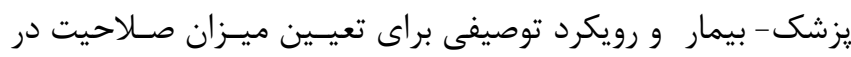

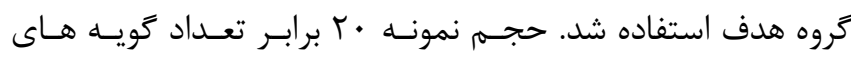

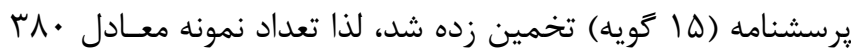

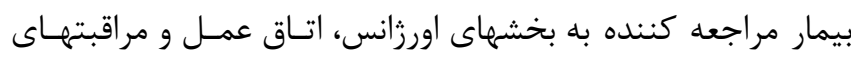

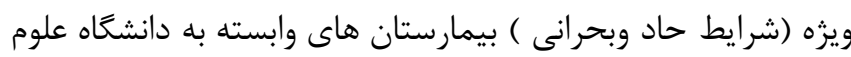

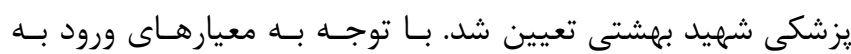

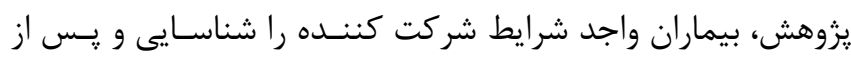

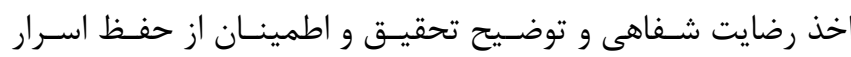

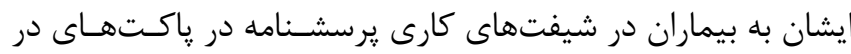

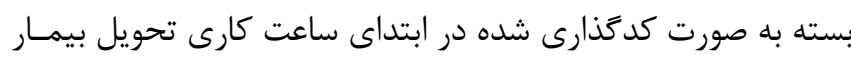

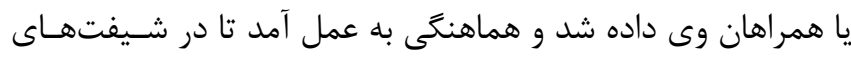

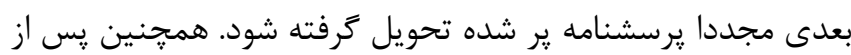

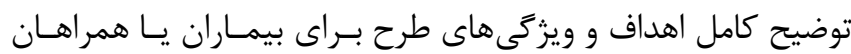

است. و- داراى مشاركت بالا در بيماران براى استفاده آسان و قابـل

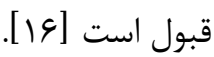

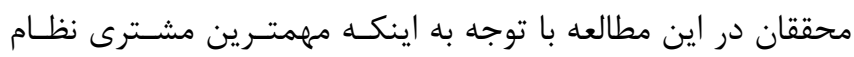

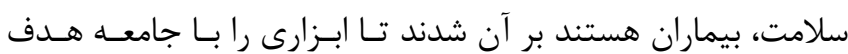

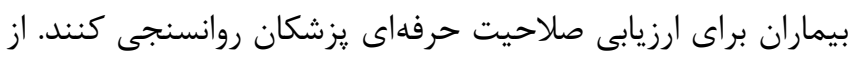

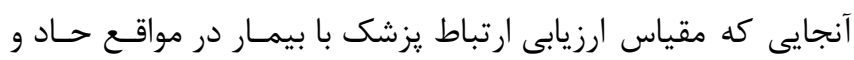

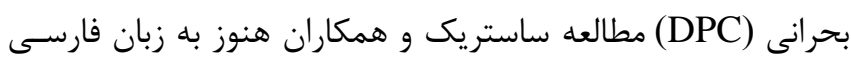

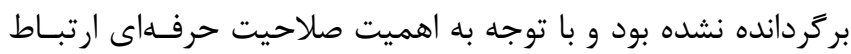

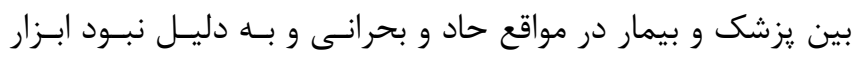

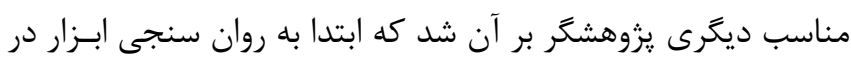

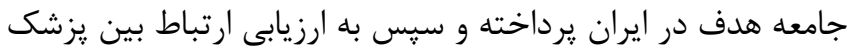
و بيمار در مواقع حاد و بحرانى بيردازد.

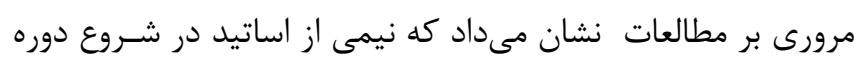

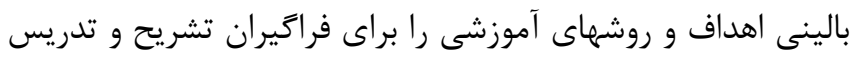

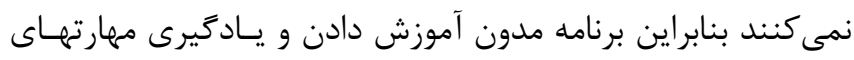

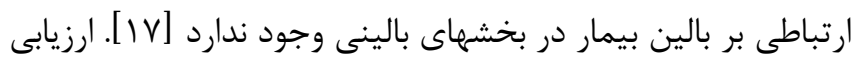

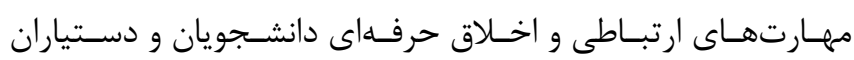

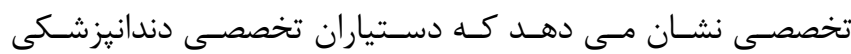

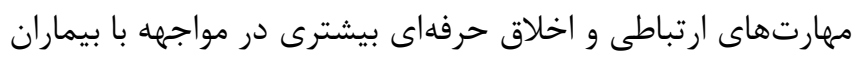

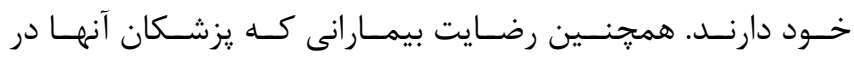

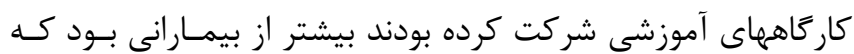

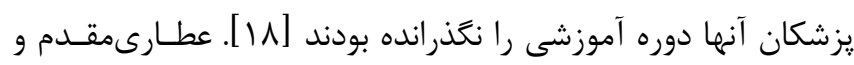

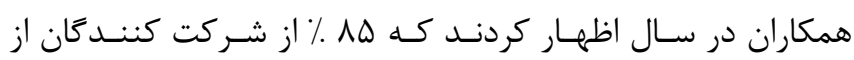

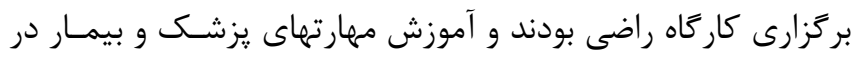

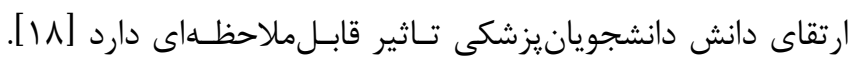

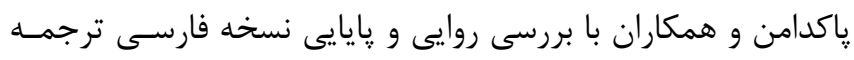

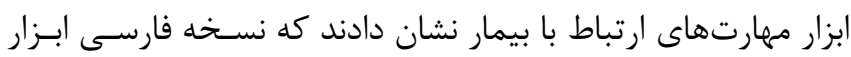

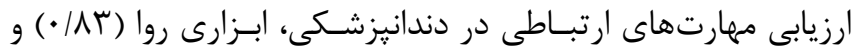

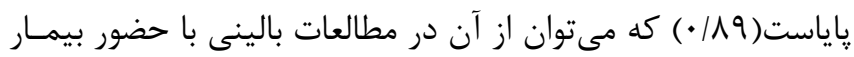

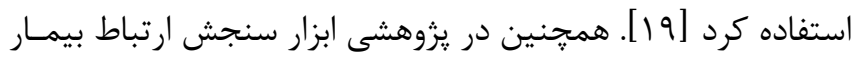

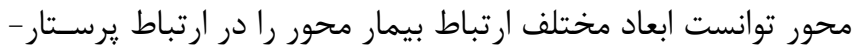

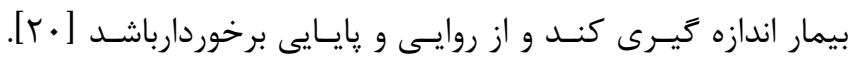

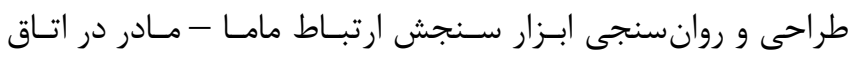

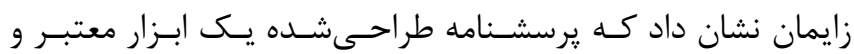

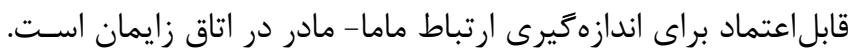

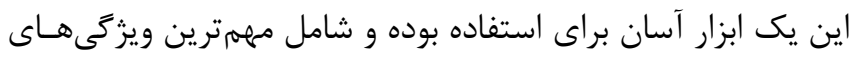


روايى سازه با استفاده از تحليل عاملى تاييدى با نرم افزار Lisrel و

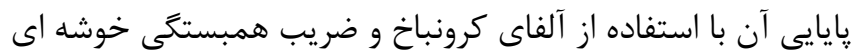

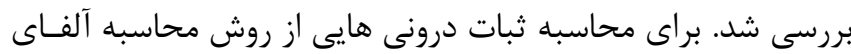
كرونباخ و روش آزمون-باز آزمون بررسى كرديد.

يافتهها

از مجموع • • نفر بيماران مراجعه كننده به بخشهاى اورزانس، اتـاق عمل و مراقبتهاى ويزه (بخـش هـاى داراى شـرايط حـاد و بحرانسى )

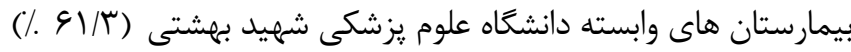

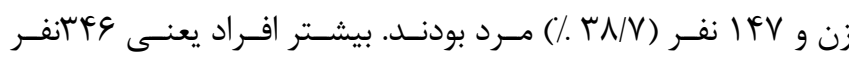

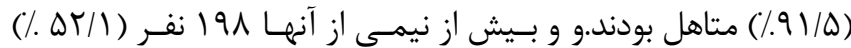

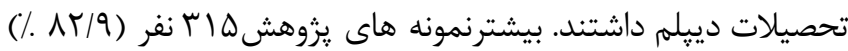

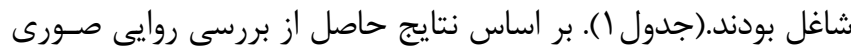
ابزار ترجمه شده توسط پانل خبركان رشـته در اولـين مرحلـه اعتبـار

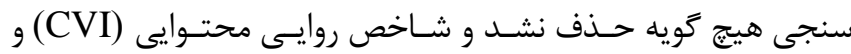

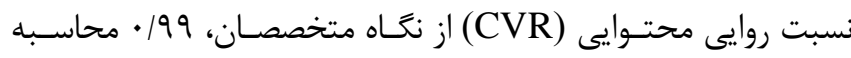

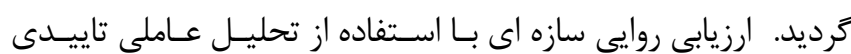
محاسبه شد. تحليل يافته ها با استفاده از نرم افزار Spss نسـخه 19 و و

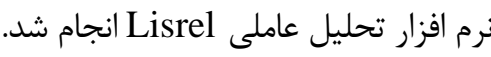
نتيجه آزمون كيسر - ماير- الكين KMO در تعيين روايى سـازه اي

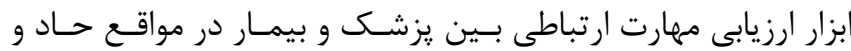

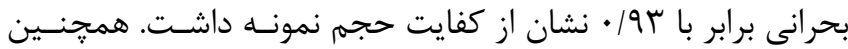

آزمون هاى بارتلت و بارتلت معنادار شدند (Pي0.000).

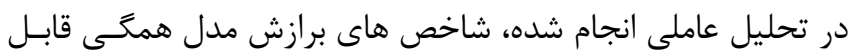
قبول بودند (جدول r). استخراج عامل ها به روش تجزيـه و تحليـل

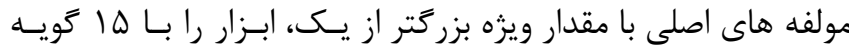

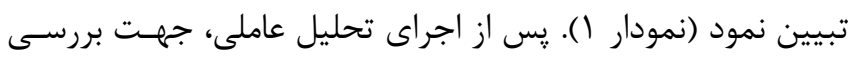

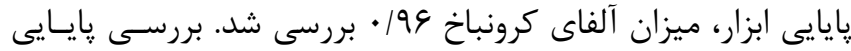

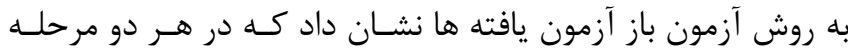

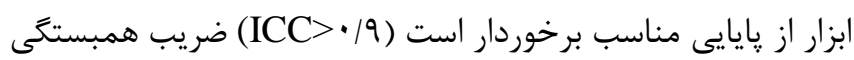

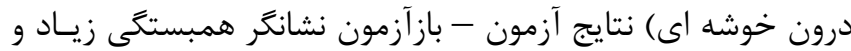
R = معنى دار بين دو مرحله آزمون و بـازازمون بـود (P<0.1)،

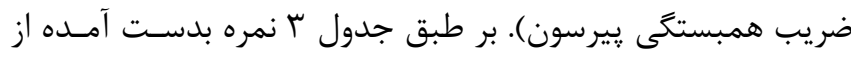

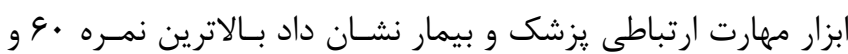
يايين ترين نمره ها است.
وى، جلب رضايت و توضيح مواردى از قبيل اختيارى بـودن شـركت

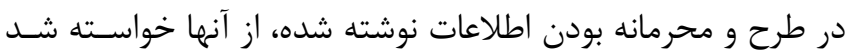

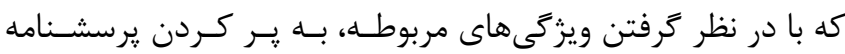

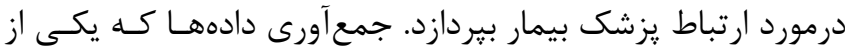

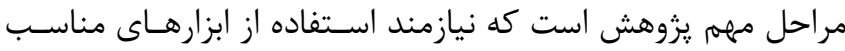

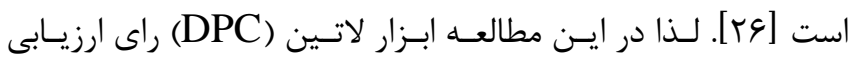

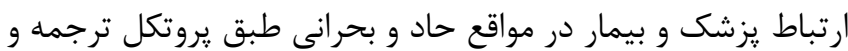

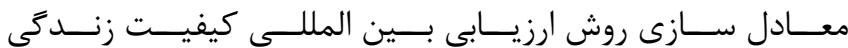
(International Quality Of Life Assessment:IQOLA)

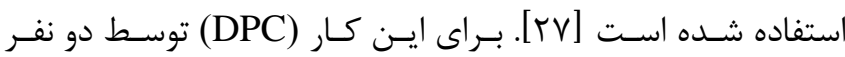

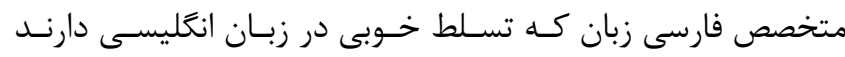

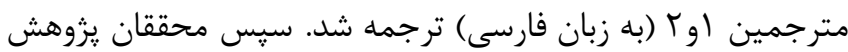

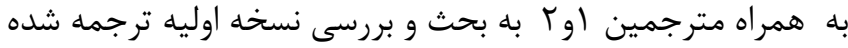

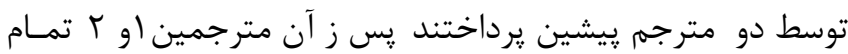

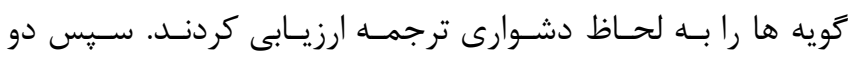

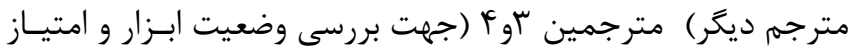

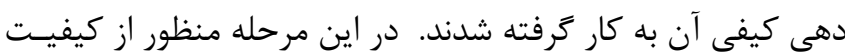

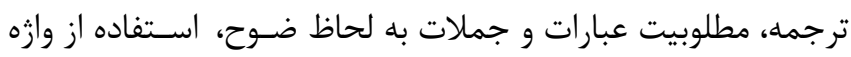

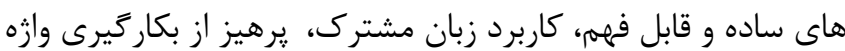

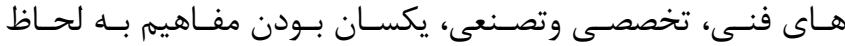

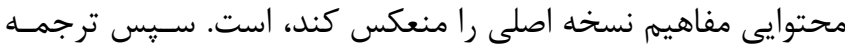
بدست آمده در مرحله قبل، توسـط دو متـرجم (متـرجمين له و و ع )

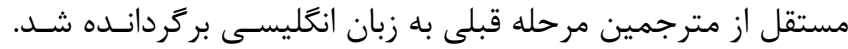

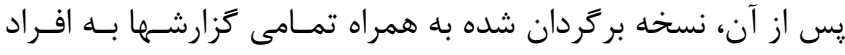

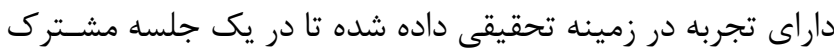

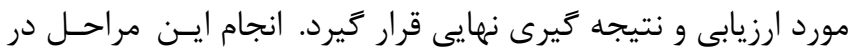

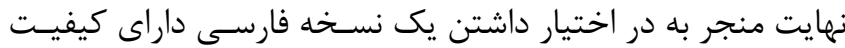

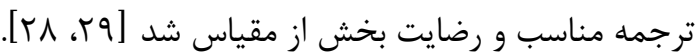

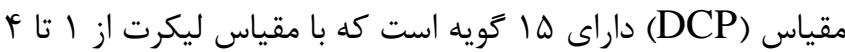

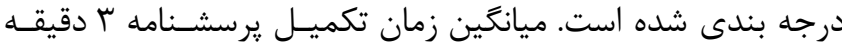

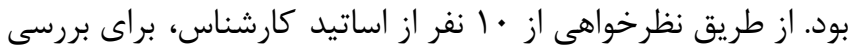

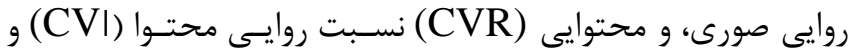

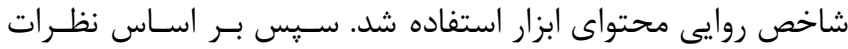

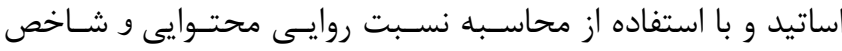

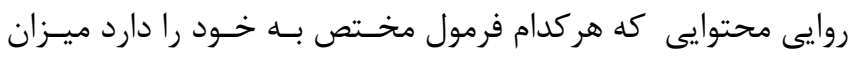
روايى محتوايى ابزار به دست آمد مدك. 
سال بيستم، شماره ينجم، مهر - آبان ..

نشريه يرؤهشكده علوم بهداشتى جهاددانشَاهى

جدول ا: مشخصات جمعيت شناسى نمونهاى يثوهش

\begin{tabular}{|c|c|c|}
\hline درصد & تعداد & \\
\hline & & جنسيت \\
\hline $91 / \pi$ & Trt l l & 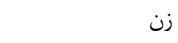 \\
\hline \multirow[t]{2}{*}{ rN/V } & $19 \mathrm{~V}$ & مرد \\
\hline & & وضعيت تاهل \\
\hline$N / \Delta$ & rr & مجرد \\
\hline \multirow[t]{2}{*}{ 9)/ब } & req & متاهل \\
\hline & & تحصيلات \\
\hline r & If. & زير دييلم \\
\hline$\Delta T / I$ & 191 & ديبلم \\
\hline$N / V$ & rז & ليسانس \\
\hline \multirow[t]{2}{*}{$r / f$} & 9 & فوق ليسانس و بالاتر \\
\hline & & وضعيت شغل \\
\hline$\Lambda T / q$ & ris & شاغل \\
\hline $\mid V / 1$ & 90 & بيكار \\
\hline rی. & & كل \\
\hline
\end{tabular}

جدول r: شاخصهاى مربوط به روايى سازه و مقادير محاسبه شده آنها

\begin{tabular}{|c|c|c|c|c|c|c|c|}
\hline RMSEA & CFI & IFI & GFI & AGFI & PNFI & CMIN/DF & \\
\hline$>\cdot 1 \cdot 1$ & $<\cdot / 9$ & $<\cdot / 9$ & $<\cdot / 9$ & $<\cdot 1 /$ & $<\cdot / \Delta$ & $\Delta-1$ & بازه قابل قبول \\
\hline .1 .1 &.$/ 90$ &.$/ 9 V$ & .199 & $\cdot 119$ & $\cdot / \Lambda$. & $9 / \pi 1$ & مقدار محاسبه شده \\
\hline دارد & دارد & دارد & دارد & دارد & دارد & دارد & وضعيت برازش \\
\hline
\end{tabular}

جدول r: شاخص هاى ابزار مهارت ارتباطى يزشك- بيمار

\begin{tabular}{|c|c|c|c|c|}
\hline بيشينه & كمينه & انحراف استاندارد & ميانكَين & ت تعداد \\
\hline $9.1 .$. & $\mid \Delta / \cdot$. & 9/91 & $F T / .9$ & TVV \\
\hline
\end{tabular}

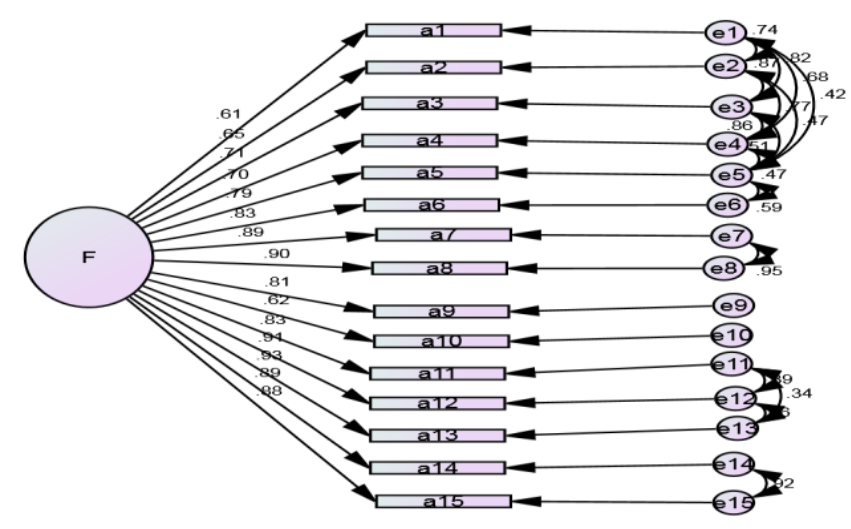

نمودار ا: تحليل عاملى تاييدى 
كرد. علاوه بر اين سازگارى داخلى ( آلفاى كرونباخ |N|•) و نمـرات

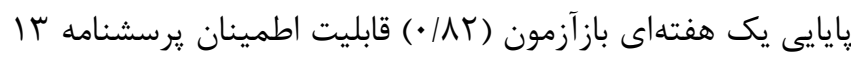

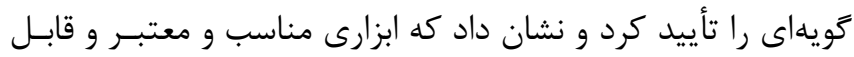

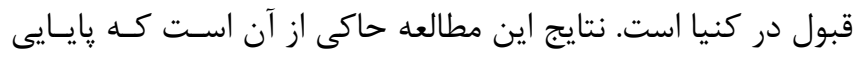

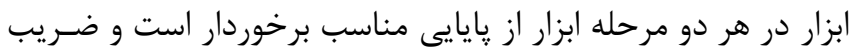

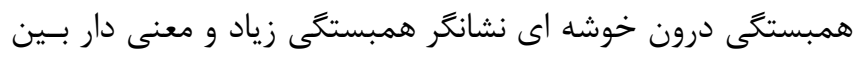

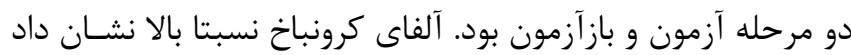
كه پايايى ابزار در حد مطلوب قرار دارد دارد. نتايج ارزيسابى روايسى سـازه

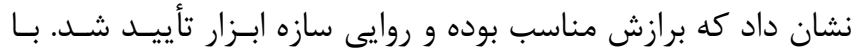
توجه به مطالب بيان شده مشخص شد كه طراحى، ترجمه و ساخت

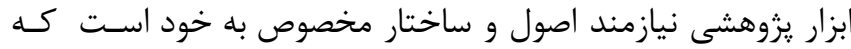

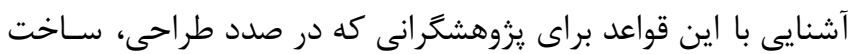

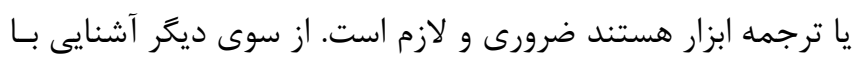

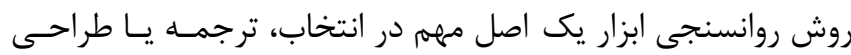
بزار است. داشتن روايى بخش مهمى از روانسنجى يكى ابزار است.

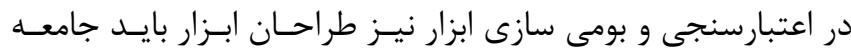

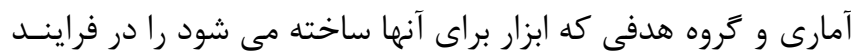

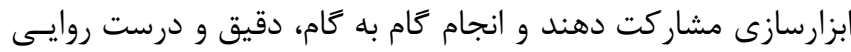
صورى و محتوا به صورت كيفى و كمى، به روايسى سـازه در مرحلـهـ دارئ.

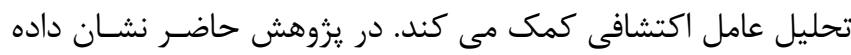
شد كه يرسشنامه از نظر روايى محتوايى و صورى و سـازه اي داراى

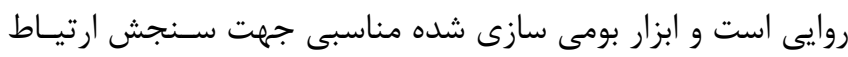
يزشك و بيمار شرايط حاد و بحرانى است.

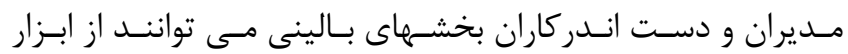

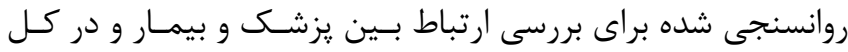

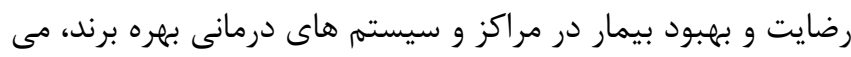

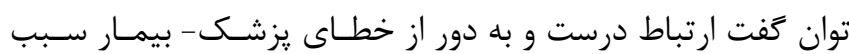

$$
\text { افزايش كيفيت زندكى در هر دو مى شود. }
$$

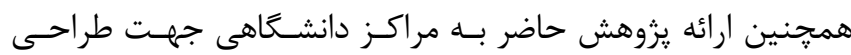

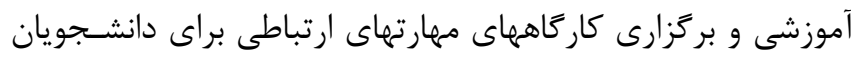

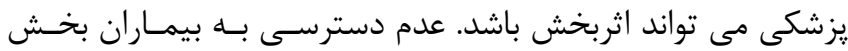

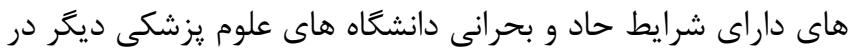

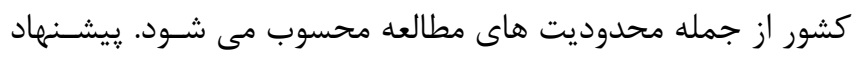

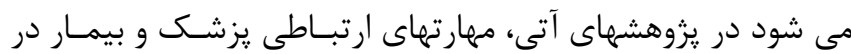

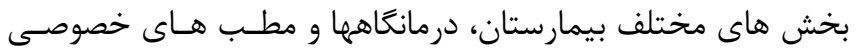

\section{بحث و نتيجه گيرى}

هدف از انجام اين يزوهش تعيين ويزَكيهاى روانسنجى ابزار (DPC)

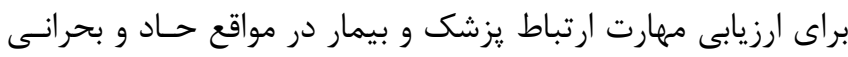
در دانشاءه علوم يزشكى شهيد بهشتى بوده است.

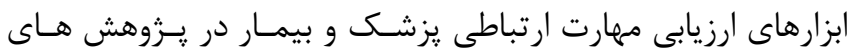
مختلف از نظر ويزگى هاى روان سنجى مورد بر رسى قرار كرفته انسد

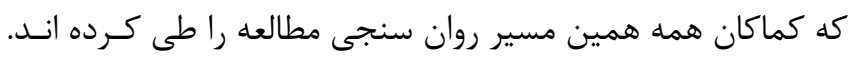

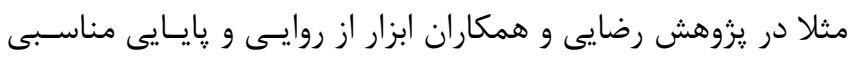

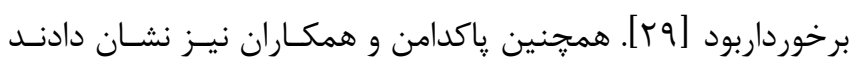

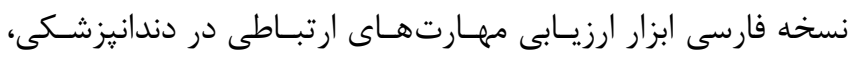

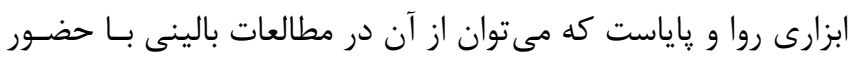

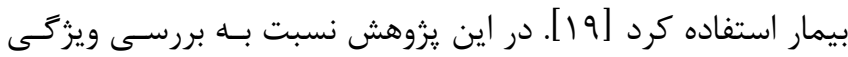

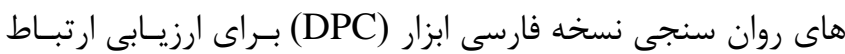

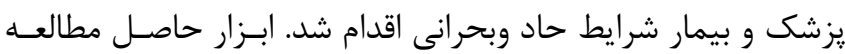
نظامند ساستر يك و همكاران بود كه در مطالعـه ايشـان نشـان داده

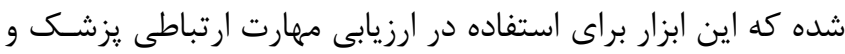

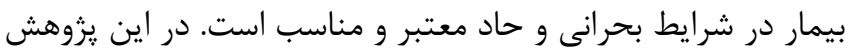

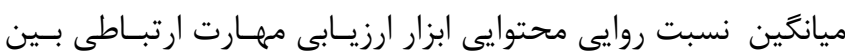

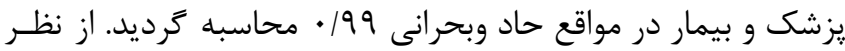

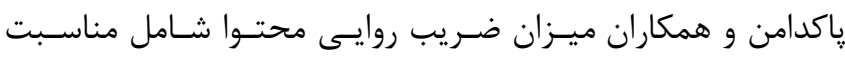

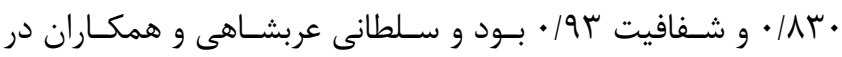

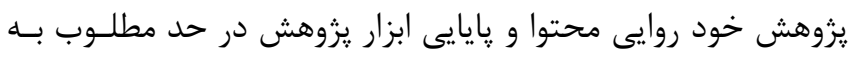

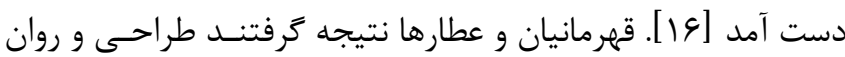

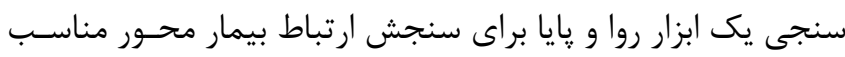

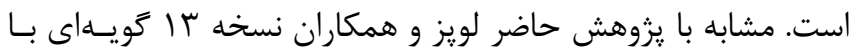

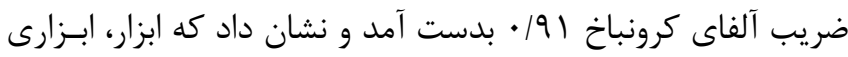

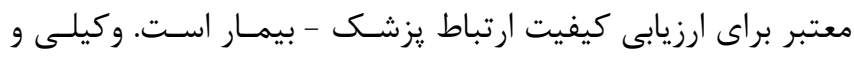

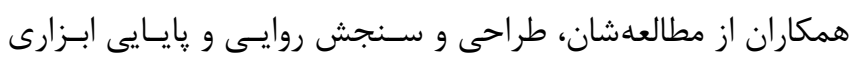

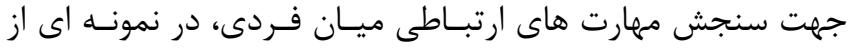

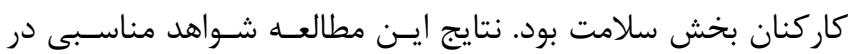

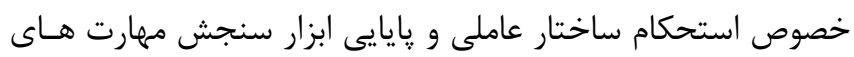

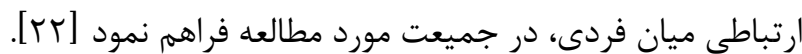

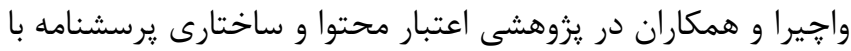

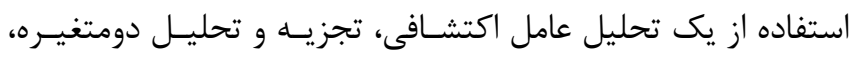

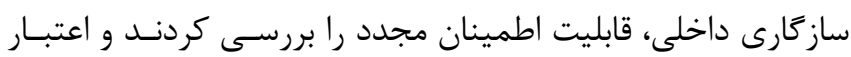

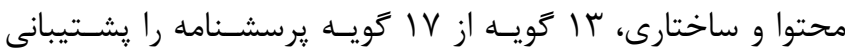




$$
\text { سياسكزاريه. }
$$

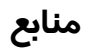

1. Gordon JA, Tancredi DN, Binder WD, WilkersonWM, Shaffer DW. Assessment of a clinical performance evaluation tool for use in a simulatorbased testing environment: a pilot study. Academic Medicine 2003;78: 45-7

2. Loge JH, Kaasa S, Hytten K. Disclosing the cancer diagnosis: the patients' experiences. European Journal of Cancer 1997;33:878-82

3. Asemani O A review of the models of physician-patient relationship and its challenges. Iranian Journal of Medical Education 2012;5:36-50

4. $\quad \mathrm{N}$ kerse SB, Mainous AG 3rd,G Young, $G$ Coster ,B Arroll , . Physician-patient relationship and medication compliance: a primary care investigation. Annals of Family Medicine 2004;2: 455-61

5. Levinson W, Pizzo PA. Patient-physician communication: it's about time. Journal of the American Medical Association 2011:4;305:1802-3

6. M shafati MJz. Physician-Patient Relationship: Ethnography of Multiple Patient Visits at Private Offices of Doctors. Journal of Qualitative Research in Health Sciences 2013;2: 46-61

7. Shafati M, Zahedi, M. A Sociological explanation of the Doctor-Patient Relationship (A qualitative study in the city of Ahvaz, Iran). Journal of Iranian Social Studies 2014; 8: 107-139 [Persian]

8. Daneshmandi M, Amiri H, Vahedi M, Farshi M, Saghafi A, Zigheymat F. Assessing the level of preparedness for confronting crisis such as flood, earthquake, fire and storm in some selected hospitals of Iran. Iranian Journal of Military Medicine 2010;12:167-71 [Persian]

9. Drennan LT, McConnell A, Stark A. Risk and crisis management in the public sector: Routledge; $2^{\text {th }}$ Edition: Thomas-Opus College of Business Minneapolis: Minnesota USA, 2014: 250-286

10. Lofgren R, Karpf M, Perman J, Higdon CM. The US health care system is in crisis: implications for academic medical centers and their missions. Academic Medicine 2006: 81: 713-20

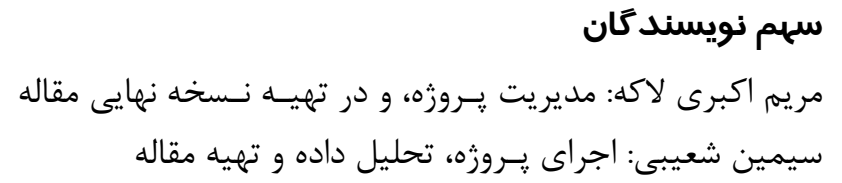

11. Suikkala A, Leino-Kilpi H. Nursing studentpatient relationship: Experiences of students and patients. Nurse Education Today 2005;25:344-54

12. Polit DF, Beck CT. The content validity index: are you sure you know what's being reported? Critique and recommendations. Research in Nursing \& Health 2006;29:489-97

13. Keen A, Klein S, Alexander DA. Assessing the communication skills of doctors in training: reliability and sources of error. Advances in Health Sciences Education 2003;8:5-16

14. Zill JM, Christalle E, Müller E, Härter M, Dirmaier J, Scholl I. Measurement of physicianpatient communication - a systematic review. PLoS One. 2014;9: 112637

15. Sustersic M, Gauchet A, Kernou A, Gibert C, Foote A, Vermorel C, et al. A scale assessing doctorpatient communication in a context of acute conditions based on a systematic review. PloS One 2018;13: 0192306

16. Soltani Arabshahi SK, Ajami A, Siabani S. Investigation of doctor-patient communication skills teaching: medical learners' perception (Stager-Intern) and staffs of Iran University of Medical Sciences \& Kermanshah University of Medical Sciences. Razi Journal of Medical Sciences 2004;11:423-31 [Persian]

17. Zamani A, Shams B, Moazzam E. Communication skills training for physicians as a strategy for enhancing patients' satisfaction: a model for continuing education. Iranian Journal of Medical Education 2004;4:15-22 [Persian]

18. Attari Moghadam J, Mokhlespour S, Valizadeh M, Momtazi S, Sharifi F, Ghodrati S, et al. Teaching "doctor-patient relationship" to the medical student: assesment of knowledge and satisfaction. Journal of Medical Education Development 2010;3:26-33 [Persian]

19. Pakdaman A, Ahmadpour R, Serajzadeh M. Dental Consultation Communication Checklist: Translation and Validation of the Persian Version. Journal of Islamic Dental Association of Iran 2015;27:173-81 [Persian] 
20. Zamanzadeh V, Ghahramanian A, Rassouli M, Abbaszadeh A, Alavi-Majd H, Nikanfar A-R. Design and implementation content validity study: development of an instrument for measuring patientcentered communication. Journal of Caring Sciences 2015;4:165 [Persian]

21. Attarha $\mathrm{M}$, Keshavarz Z, Bakhtiari M, Jamilian M, Zayeri F. Development and Psychometric Tests of Midwife-Mother Relationship Scale (MMRS) in Delivery Room. Global Journal of Health Science 2017;9:184 [Persian]

22. Vakili MM, Hidarnia AR, Niknami S. Development and psychometrics of an interpersonal communication skills scale (ASMA) among Zanjan Health volunteers. Journal of Hayat 2012;18:5-19 [Persian]

23. López PR. Psychometric validation of the Spanish version of the Patient-Doctor Relationship Questionnaire (PDRQ). Actas Españolas de Psiquiatría 2009;37:94-100

24. Wachira J, Middlestadt S, Reece M, Peng CYJ, Braitstein P. Psychometric assessment of a physician-patient communication behaviors scale: the perspective of adult HIV patients in Kenya. AIDS Research and Treatment 2013;2013:706191

25. Campbell C, Lockyer J, Laidlaw T, MacLeod H. Assessment of a matched-pair instrument to examine doctor- patient communication skills in practising doctors. Medical Education 2007;41:123
26. Afrasiabifar A., Yaghmaei F, Abdoli S, Abed Saeidi ZH. Research Tool translation and cross cultural adaptation. Advances in Nursing and Midwifery (Faculty of Nursing of Midwifery Quartely) 2006;16:58-67[Persian]

27. Bullinger $\mathrm{M}$, Alonso $\mathrm{J}$, Apolone $\mathrm{G}$, Leplege A, Sullivan M, Wood-Dauphinee S, et al. Translating health status questionnaires and evaluating their quality: the IQOLA Project approach. International Quality of Life Assessment. Journal of Clinical Epidemiology1998;51:913-23

28. Taghizadeh Z, Ebadi A, Montazeri A, Shahvari Z, Tavousi M, Bagherzadeh R. Psychometric properties of health related measures. Part 1 : Translation, development, and content and face validity. Payesh (Health Monitor) 2017;16:34357[Persian]

29. Aaronson NK, Acquadro C, Alonso J, Apolone G, Bucquet D, Bullinger M, Bungay $\mathrm{K}$, Fukuhara S, Gandek B, Keller S, et al. International Quality of Life Assessment (IQOLA) Project. Quality Life Research 1992;1:349-51 
يقيوست: مقياس (DPC) جهت ارزيابى مهارت ارتباط بزشك و بيمار در شرايط بحرانى و حاد

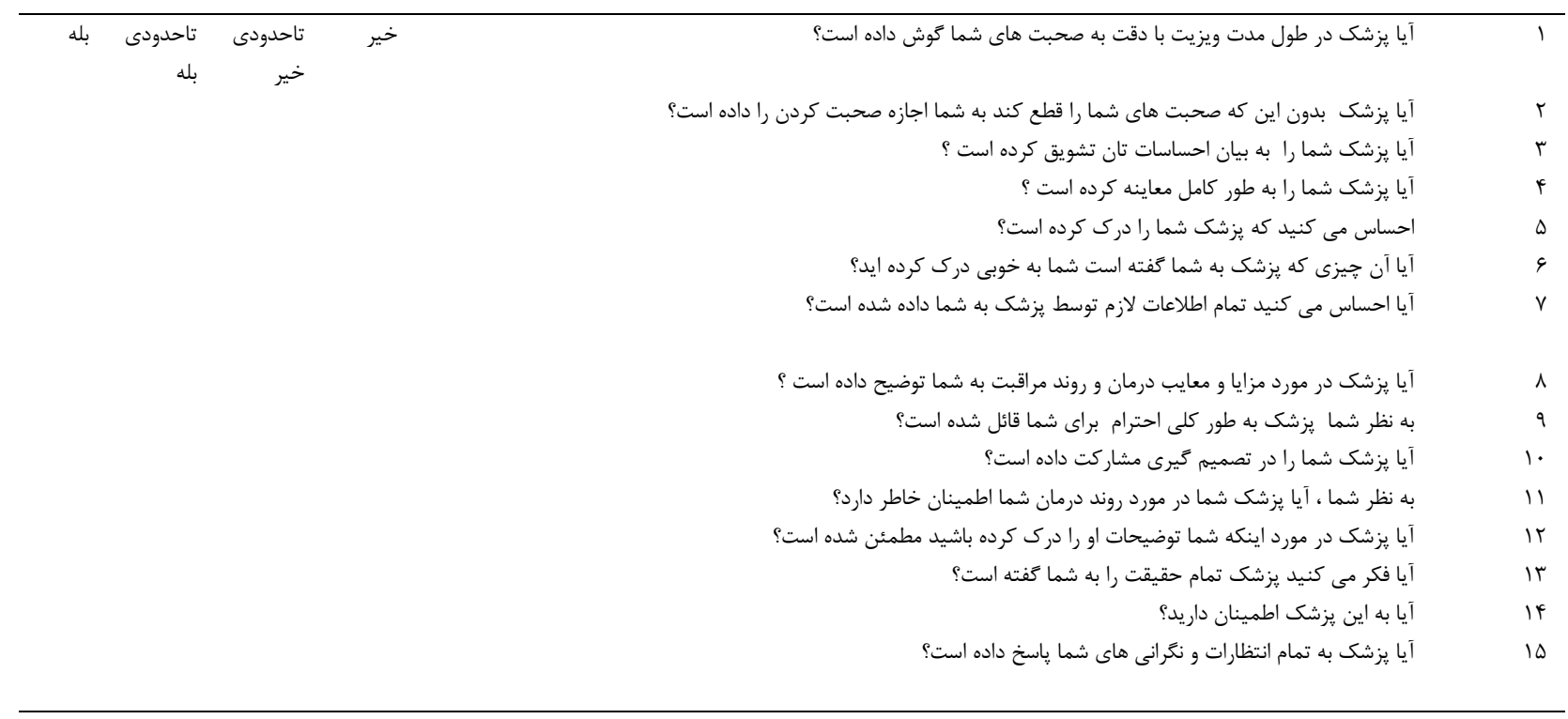

